\title{
Status of etoricoxib in the treatment of rheumatic diseases. Expert panel opinion
}

\section{Brygida Kwiatkowska ${ }^{1}$, Maria Majdan², Agnieszka Mastalerz-Migas ${ }^{3}$, Maciej Niewada ${ }^{4}$, Barbara Skrzydło-Radomańska ${ }^{5}$, Artur Mamcarz ${ }^{6}$}

${ }^{1}$ Early Arthritis Clinic National Institute of Geriatrics, Rheumatology and Rehabilitation, Warsaw, Poland

${ }^{2}$ Department of Rheumatology, Medical University in Lublin, Poland

${ }^{3}$ Department of Family Medicine, Wroclaw Medical University, Poland

${ }^{4}$ Chair and Department of Experimental and Clinical Pharmacology, Medical University of Warsaw, Poland

${ }^{5}$ Chair and Department of Gastroenterology, Medical University in Lublin, Poland

${ }^{6} 3^{\text {rd }}$ Department of Internal Disease and Cardiology, Medical University of Warsaw, Poland

\begin{abstract}
Pain is one of the most disabling symptoms of rheumatoid diseases. Patients with pain secondary to osteoarthritis (OA), rheumatoid arthritis (RA), ankylosing spondylitis (AS) or gout require effective analgesic treatment, and the physician's task is to select a drug that is best suited for an individual patient. The choice of pharmacotherapy should be based both on drug potency and clinical efficacy, and its safety profile, particularly in the elderly population, as the number of comorbidities (and hence the risk of treatment complications and drug interactions) rises with age. In cases involving a high risk of gastrointestinal complications or concerns about hepatotoxicity, with a low cardiovascular risk, the first-line nonsteroidal anti-inflammatory drugs to consider should be coxibs including etoricoxib.
\end{abstract}

Key words: etoricoxib, coxibs, cyclooxygenase COX-2, pain treatment, osteoarthritis, rheumatoid arthritis, ankylosing spondylitis, gout.

\section{Introduction}

One of the main symptoms accompanying the majority of rheumatic diseases is pain. This is why a non-steroidal anti-inflammatory drug (NSAID) is prescribed by rheumatologists or primary care physicians to most patients with rheumatoid diseases. Etoricoxib belongs to the class of non-steroidal anti-inflammatory drugs. It is an oral selective inhibitor of cyclooxygenase-2 (COX-2). Cyclooxygenase is an enzyme responsible for the production of prostaglandins. It occurs as two isoenzymes: COX-1 and COX-2. COX-2 is an inflammation-induced isoenzyme responsible for the synthesis of prostanoid mediators of pain, inflammation and fever. Pharmacological studies show that etoricoxib, similarly to other coxibs, causes dose-dependent inhibition of COX-2 ac- tivity, without affecting COX-1 activity. Based on clinical trial results, etoricoxib was approved for the treatment of osteoarthritis, rheumatoid arthritis, ankylosing spondylitis and pain caused by gout attack.

At present, treatment with coxibs is a relatively uncommon therapeutic option mainly because of concerns about their safety which arose when rofecoxib was withdrawn from the market in 2004. However, every medicine of the coxib family (rofecoxib, celecoxib, valdecoxib, etoricoxib, lumiracoxib) has different pharmacokinetic and pharmacodynamic parameters, which translates into differences in biological activity, clinical efficacy and safety profile. This is due to relatively significant differences in the structure of drugs of the coxib class. For example, etoricoxib contains a sulfonyl group, and celecoxib and valdecoxib have a sulfonamide group responsible 
for the development of allergic reactions. Comparing etoricoxib with other representatives of the coxib class, a notable observation is that the time required for the drug to reach maximum concentration in the blood is short ( $T_{\max }=1$ hour). As a result, etoricoxib is effective in controlling acute pain [1]. In contrast, the $T_{\max }$ of celecoxib is $2-3$ hours.

\section{Etoricoxib in the treatment of osteoarthritis}

Osteoarthritis (OA) is a disease involving a combination of biological (including inflammatory) and mechanical factors which destabilize the equilibrium of articular cartilage and subchondral bone layer degradation and synthesis processes. As a result, the articular cartilaginous tissue becomes soft and fibrillose, and ulcers and cartilage loss are observed. The subchondral bone layer is thickened and indurated, and osteophytes and subchondral cysts are formed.

Osteoarthritis can be classified into either primary (having no identifiable underlying cause) or secondary (arising from local injuries and/or abnormalities in joint structure or comorbidities). Some forms of OA, such as erosive hand osteoarthritis, have a strong genetic background, and the presence of this form of the disease increases the likelihood of coexisting osteoarthritis of the knees [2, 3].

The incidence of osteoarthritis in the general European population over 40 years of age is currently growing. This is due to the growth of obesity, which is an important risk factor for osteoarthritis, and the ageing of societies accompanied by an increase in average life expectancy. Despite advances in medicine, there are currently no options for effective conservative treatment of osteoarthritis or strategies for its successful prevention. Consequently, OA is treated symptomatically, and therapy is aimed at relieving pain and improving the quality of life of patients. In order to achieve this target, long-term pharmacotherapy is indicated to prevent pain from occurring or worsening. Therefore, it is crucial to monitor pain experienced by the patient over 24 hours and select medications that ensure either complete pain elimination or maximum reduction.

According to the American College of Rheumatology (ACR) 2012 recommendations, hand osteoarthritis should be treated with topical capsaicin, topical NSAIDs, oral NSAIDs (including COX-2 selective inhibitors) and, if the above therapeutic modalities prove ineffective, with tramadol. In patients with hip osteoarthritis, the ACR recommends paracetamol, oral NSAIDs, tramadol and intraarticular glucocorticosteroid injections [4]. In 2011 a panel of European experts announced recommenda- tions for the use of NSAIDs in rheumatic diseases [5]. Before the introduction of NSAIDs, the experts recommend taking a patient's history of upper gastrointestinal events and evaluating the risk of cardiovascular complications based on the HeartScore algorithm of the European Society of Cardiology. Patients at a high risk of gastrointestinal complications should be additionally assessed for a history of treatment with low doses of acetylsalicylic acid, anticoagulants or other antiaggregation drugs, and therapy with glucocorticosteroids in general.

However, pharmacological treatment of osteoarthritis is challenging on account of several factors: (1) paracetamol at a dose of $>2 \mathrm{~g} /$ day, as recommended by the ACR, increases the risk of gastrointestinal complications, particularly in patients over 65 years of age, with a history of prior gastrointestinal incidents or in patients $>85$ years of age [6]; (2) topical treatment is not always possible, as some joints (such as the hip joint) are poorly accessible through the skin; (3) the opioid tramadol is associated with a risk of dependence (especially when used for more than 12 months), and in patients $>75$ years of age the maximum daily dose should not exceed $400 \mathrm{mg}$; hence it is a serious therapeutic error to introduce tramadol as the first analgesic drug (according to applicable guidelines, it should be at the top step of the analgesic ladder) [7]; (4) glucocorticosteroids have very limited indications and carry a large risk of adverse effects including cardiovascular risk. A relatively safe and effective analgesic option in patients with osteoarthritis seems to be etoricoxib. A comparative analysis evaluating treatment with etoricoxib (30 mg/day), ibuprofen (2,400 mg/day) and placebo in patients with osteoarthritis showed etoricoxib and ibuprofen to have superior efficacy to placebo. However, etoricoxib exhibited a higher efficacy in the relief of night pain than ibuprofen. Furthermore, the group treated with etoricoxib had a much lower incidence of gastrointestinal adverse effects [8]. In a different study, etoricoxib at a dose of $30 \mathrm{mg} /$ day had comparable safety and efficacy to celecoxib at a dose of $200 \mathrm{mg} /$ day, and a better safety and efficacy profile than placebo in patients with knee and hip osteoarthritis [9].

The Etoricoxib versus Diclofenac Sodium Gastrointestinal Tolerability and Effectiveness (EDGE) study showed that etoricoxib at a dose of $90 \mathrm{mg} /$ day was safer than diclofenac at a dose of $150 \mathrm{mg} /$ day (the rate of cumulative discontinuation due to gastrointestinal adverse effects was 9.4 per 100 patient-years (PY) for etoricoxib vs. 19.2 per 100 PY for diclofenac). The risk of thromboembolic cardiovascular events was similar for etoricoxib and diclofenac (1.25 vs 1.15 events per 100 PY). The risk of treatment discontinuation on account of hy- 
pertension was higher for etoricoxib (2.3\%) than for diclofenac (0.7\%) [10].

Another comparative study analyzed the efficacy and safety of one-year treatment with etoricoxib at doses of 30, 60 and $90 \mathrm{mg} /$ day vs. diclofenac at a dose of $150 \mathrm{mg} /$ day. The efficacy of etoricoxib was similar to that of diclofenac, but etoricoxib was associated with a lower incidence of adverse gastrointestinal effects than diclofenac (13.1, 14.7 and 13.5\% for etoricoxib at doses of 30,60 , and $90 \mathrm{mg}$, respectively, compared to $22.5 \%$ for diclofenac). Also, more patients in the diclofenac group discontinued treatment because of all adverse effects combined $(11.8 \%)$ than in the group treated with the highest dose of etoricoxib (90 mg/day, 6.8\%) [11]. High analgesic efficacy of etoricoxib in osteoarthritis, compared to other NSAIDs, was also demonstrated in a 2012 study reported in The Open Rheumatology Journal. Etoricoxib was shown to provide more effective relief of pain and improvement in joint function than paracetamol, diclofenac, naproxen, ibuprofen, celecoxib and lumiracoxib [12]. The most recent and most extensive meta-analysis comparing the efficacy of various NSAIDs in the treatment of pain secondary to knee and hip osteoarthritis was published in The Lancet in 2016. The analysis was based on the findings of 74 randomized clinical trials with a total of 58,556 patients. On the basis of the available data, etoricoxib at $60 \mathrm{mg}$ and diclofenac at $150 \mathrm{mg}$ were shown to be the most effective drugs available for the treatment of pain in patients with knee or hip osteoarthritis. Diclofenac 150 mg demonstrated the highest efficacy in terms of motor function improvement [13].

\section{Etoricoxib in the treatment of rheumatoid arthritis}

Rheumatoid arthritis (RA) is a chronic autoimmune inflammatory disease causing joint destruction and organ damage. The most characteristic features of RA include pain, stiffness and edema of the hands and feet, although inflammation may also affect other joints. RA occurs in serologically positive or negative forms, which refers to the presence or absence of the rheumatoid factor (RF) IgM-class antibodies or anti-CCP antibodies (against cyclic citrullinated peptide) in the serum. The incidence of rheumatoid arthritis in the general population is estimated at $0.3-1.5 \%$ (in Europe $-0.8 \%$, in Poland $-0.9 \%$ ). Women are affected three times as often as men, and the disease most commonly starts between 40 and 50 years of age. According to the current EULAR recommendations, the treatment of patients with RA should aim at the best care and be based on a shared decision between the patient and the rheumatologist.
Therapeutic decisions should be made depending on the evaluation of disease activity and other factors, such as progression of structural damage, comorbidities and safety of treatment. Rheumatologists are the specialists who should primarily care for patients with RA, and therapy should be selected taking into consideration the high individual and societal costs generated by RA [14]. Treatment should be aimed at reaching the target of sustained remission or low disease activity. Consequently, therapy with disease-modifying antirheumatic drugs (DMARDs) should be started as soon as the diagnosis of RA is made. The first-line drug is methotrexate. Patients with a contraindication to methotrexate should be treated with leflunomide or sulphasalazine. When starting methotrexate treatment, low doses of glucocorticosteroids (GC) should be used, but the duration of GC therapy should be as short as possible, not exceeding 6 months. Disease activity requires frequent monitoring (every 1-3 months), and if there is no improvement by 3 months after the start of treatment or the therapeutic target (remission or low disease activity) has not been reached by 6 months, the treatment regimen should be changed.

Despite treatment, patients with rheumatoid arthritis may occasionally develop pain or their pre-existing pain may be aggravated. In accordance with the recommendations, such patients should take paracetamol as the first-line drug. If paracetamol is ineffective, patients should be switched to another NSAID, suitable in terms of safety and efficacy. In such cases, a beneficial effect in some patients can be achieved by adding etoricoxib to the primary regimen of disease-modifying treatment, since this management strategy significantly reduces pain and improves daily functioning of the patient [1517]. The safety profile of etoricoxib in patients with RA is better than that of diclofenac, which was demonstrated in the randomized clinical study EDGE-II [18].

\section{Etoricoxib in the treatment of ankylosing spondylitis}

Ankylosing spondylitis (AS) is a chronic progressive inflammatory disease classified in the axial spondyloarthritis disease family and involving primarily sacroiliac joints, small facet joints and spinal ligaments, causing their gradual stiffening. The disease may run a mild course leading to spontaneous remissions lasting many years or a severe progressive course culminating in serious abnormalities of the motor system within a short period of time.

The pathogenesis of AS has not yet been established. Contributing factors are numerous and include genetic predisposition (familial association, frequent presence 
of HLA B-27 antigen), and immune, infectious and other environmental factors. The incidence of AS shows considerable global variation. In Central Europe, the disease affects approximately $0.3-0.5 \%$ of the population. AS is more common in men than in women. The onset is usually between the ages of 17 and 35. As a rule, the first manifestation is the so-called inflammatory spinal pain in the lumbosacral region. Characteristically, pain associated with AS does not improve with rest. It worsens in the second half of the night, often waking the patient from sleep, and tends to resolve gradually during the day with physical activity. Alternating buttock pain is also a common sign. In addition to spinal changes, AS is also accompanied by enthesitis and possible involvement of peripheral joints, organs and systems (uveitis, inflammatory bowel disease, psoriasis and cardiovascular abnormalities).

According to the most recent ASAS-EULAR recommendations, axial spondyloarthritis is a potentially severe disease with diverse clinical manifestations, requiring multidisciplinary management coordinated by the rheumatologist. The primary goals of therapy should be to improve the quality of life of patients, control symptoms and inflammation, prevent progressive structural damage, and normalize function and social participation of patients. Optimal treatment of AS should consist of non-pharmacological and pharmacological methods, and should be based on a shared decision between the patient and the rheumatologist. Therapy should be individualized according to the existing (axial, peripheral, extra-articular) manifestations. First-line treatment in patients with ankylosing spondylitis is based on non-steroidal antiinflammatory drugs. NSAIDs should be administered chronically at maximum approved or maximum tolerated doses because they have been shown to act not only as analgesic agents but also as disease-modifying drugs, reducing the activity of the disease and inhibiting the progression of radiological changes. Furthermore, long-term treatment with NSAIDs in spondyloarthropathies may reduce the risk of cardiovascular complications associated with these diseases, and continued NSAID therapy in patients treated with tumor necrosis factor $\alpha$ (TNF- $\alpha$ ) inhibitors improves the efficacy of biological treatment [19-23]. Consequently, patients whose condition improves after NSAIDs are advised to continue treatment even after the regression of symptoms. Analgesics such as paracetamol and opiates might be considered for pain management if first-line treatment with NSAIDs is ineffective, contraindicated and/or poorly tolerated. In addition to pharmacological treatment, patients should be encouraged to follow a regular exercise routine.
One of the best NSAID options currently available in patients with AS is etoricoxib. A comparative study evaluating a year's treatment with etoricoxib and naproxen in patients with AS demonstrated superior therapeutic efficacy of etoricoxib used at $90 \mathrm{mg} /$ day. In addition, etoricoxib was found to have a favorable safety profile and good tolerability [24]. High analgesic effectiveness of etoricoxib in patients with AS was also demonstrated in a meta-analysis of 26 clinical trials involving a total of 3,410 participants treated with 20 different NSAIDs for 2-12 weeks [25]. Etoricoxib was shown to be the most effective drug in terms of reducing pain. In another study etoricoxib was found to be effective in the treatment of patients with AS refractory to other, traditional NSAIDs [26]. An important fact to consider is that AS tends to coexists with inflammatory bowel disease (Crohn's disease, ulcerative colitis) in $5-10 \%$ of cases. In this group of patients classic NSAIDs usually cause exacerbation of inflammatory bowel disease, which constitutes a contraindication to their use. Etoricoxib is not known to exacerbate inflammatory bowel disease in these patients, and the incidence of adverse effects associated with etoricoxib is not statistically significantly different from placebo [27-29].

In addition to individual patient benefits, the use of etoricoxib in AS therapy is also validated for pharmacoeconomic reasons. A study published in 2010 in PharmacoEconomics, involving patients treated with etoricoxib, celecoxib and non-selective NSAIDs, assessed a number of indices including BASFI, BASDAI and QALYs, and costs of treating adverse effects. The expected savings associated with etoricoxib were found to be $£ 13,620$ compared to celecoxib (200/400 mg), $£$ 9,957 compared to diclofenac, and $£$ 9,863 compared to naproxen [30].

\section{Etoricoxib in the management of acute gout pain}

A factor contributing to the pathogenesis of gout is hyperuricemia, which is one of the most common metabolic disorders affecting humans. Hyperuricemia affects one in four men and one in ten women in contemporary high-tech societies. It is estimated that about $20 \%$ of people with hyperuricemia develop gout, a disease characterized by alternating attacks of acute arthritis and periods of chronic polyarthritis. Arthritis is accompanied by the deposition of uric acid crystals in various body tissues and organs, and kidney damage (with symptoms of damage to the tubulointerstitial tissue and kidney vessels, and glomerular dysfunction). Over the last two decades, the incidence of gout has doubled, and at present the disease occurs in $1-2 \%$ of the population. The 
incidence of gout clearly rises with age. It affects $6 \%$ of men in the $6^{\text {th }}$ decade of life and the rate grows to $10 \%$ of men between the $6^{\text {th }}$ and 8 th decade. Pain accompanying acute arthritis secondary to gout is described as one of the most severe pains known in medicine. An acute gout attack usually begins suddenly, without warning. It may be triggered by a minor injury, consumption of a large meal with alcohol, dehydration, fatigue, infection or surgery. The first symptom of acute arthritis is severe pain with edema and redness in the affected joint(s), usually beginning at night. An elevated body temperature may also be present during the attack, and high levels of acute inflammation markers (leukocytosis, ESR, CRP, fibrinogen) are noted. As a rule, a gout attack resolves spontaneously after 7-10 days, but the pain experienced by the patient is very severe and debilitating. Many patients experiencing such an occurrence report to the $A \& E$ and/or emergency orthopedic department. According to the current ACR and EULAR recommendations the therapy of gout follows the treat-to-target approach, and it is aimed at: (1) elimination of pain; (2) prevention of organ damage caused by the deposition of uric acid crystals [31-34]. NSAIDs have a special role in the treatment of gout. They should be used not only during, but also between, acute attacks, during uric acid-lowering treatment, in order to prevent gout attacks.

A review of findings obtained in 22 studies analyzing the use of various NSAIDs during acute gout shows that selective and non-selective COX-2 inhibitors have similar efficacy, but selective inhibitors produce significantly fewer adverse effects [35]. Another systematic review of the literature found that etoricoxib produced an analgesic and antiinflammatory effect similar to indometacin, diclofenac and naproxen (both in subjective patient assessment and objective physician evaluation) and, in addition, it had a lower incidence of adverse events [36]. This is important because a major concern in the treatment of gout is the safety of long-term NSAID therapy, especially in view of the fact that gout frequently coexists with metabolic syndrome and in the elderly population it is associated with multiple morbidities.

\section{Gastrointestinal safety of etoricoxib}

Gastrointestinal (GI) effects rank among the most common side effects of NSAID therapy [37]. Gl events include dyspepsia (affecting 15-25\% of patients treated with NSAIDs), peptic ulcers (15-30\% of patients taking NSAIDs), and GI perforations and bleeding (their risk increases 2-6 times in patients using NSAIDs). Bleeding is attributable to an imbalance between aggressive factors affecting the gastrointestinal mucosa and protective factors such as prostaglandins, the synthesis of which is inhibited by NSAIDs acting non-selectively on constitutive cyclooxygenase (COX-1). Gastrointestinal bleeding is a serious complication of NSAID treatment ( $6 \%$ of Gl hemorrhages are fatal); however, unfortunately in many cases patients exhibit no signs suggesting a possible development of the complication. As a result, physicians should screen patients for risk factors, the most important of which include a history of peptic ulcer disease, especially complicated by bleeding, and $H$. pylori infection [38]. Consequently, H. pylori eradication and monitoring of the success of eradication are necessary before the initiation of NSAID treatment. Evaluation of gastrointestinal risk should also involve the following factors:

- dose of NSAIDs used,

- concomitant use of ASA,

- concomitant use of anticoagulants,

- concomitant treatment with glucocorticoids,

- patient age (age over 60 years is a factor for increased risk),

- alcohol use,

- severe comorbidities [39-41].

On account of the high risk of gastrointestinal adverse effects, chronic NSAID therapy requires the use of gastroprotective agents [42]. The most common gastroprotective drugs are proton pump inhibitors (PPI). They are effective in protecting the upper section of the gastrointestinal tract, but they may have deleterious effects on the lower gastrointestinal tract. The use of probiotics has potential to reduce the adverse effect of NSAIDs on the lower section of the GI tract.

The safety of etoricoxib and its effects on the gastrointestinal tract were studied in the randomized clinical trials EDGE, EDGE II and MEDAL. In the studies, etoricoxib was compared with diclofenac. The EDGE trial enrolled 7,111 patients with osteoarthritis (OA) who were treated with etoricoxib at a dose of $90 \mathrm{mg} /$ day (exceeding 1.5 times the dose indicated for OA) or diclofenac at $150 \mathrm{mg} /$ day for an average of 9.1 months (maximum 16.6 months, median 11.4 months). EDGE II was a trial involving 4,086 patients with RA who took etoricoxib at a dose of $90 \mathrm{mg} /$ day or diclofenac at a dose of $150 \mathrm{mg} /$ day for an average of 19.2 months (maximum 33.1 months, median 24 months). The MEDAL trial enrolled 17,804 patients with OA and 5,700 patients with RA who took etoricoxib at a dose of $60 \mathrm{mg}(\mathrm{OA})$ or $90 \mathrm{mg}$ (OA and RA) or diclofenac at $150 \mathrm{mg} /$ day for an average period of 20.3 months (maximum 42.3 months, median 21.3 months). The three trials (EDGE, EDGE ॥ and MEDAL) constituted the pooled MEDAL program. In each of the constituent trials, a significantly lower rate of treatment discontinuation due to any clinical gastrointestinal adverse effects (e.g. dyspepsia, abdominal pain, ulcers) was observed in the group treated with etoricoxib than 
in the group treated with diclofenac. The rates of treatment discontinuation due to any clinical gastrointestinal adverse effects per 100 patient-years throughout the entire period of study were: 3.92 for etoricoxib and 5.69 for diclofenac in MEDAL, 9.12 for etoricoxib and 12.28 for diclofenac in EDGE, and 5.0 for etoricoxib and 7.2 for diclofenac in the EDGE II trial [43].

Another study assessed the incidence of endoscopically detected gastric and duodenal ulcers ( $\geq 3 \mathrm{~mm}$ in size) in patients taking placebo, etoricoxib at a dose of $120 \mathrm{mg} /$ day or ibuprofen at a dose of 2,400 mg/day for 6 and 12 weeks. The incidence of peptic ulcers was found to be statistically significantly lower in the group of patients treated with etoricoxib than in the ibuprofen group [44]. The finding is important, since ibuprofen is very commonly used by patients with peptic ulcers because of its OTC status, and it is considered by most patients as completely safe.

The MEDAL trial program also compared etoricoxib and diclofenac in terms of hepatotoxicity. Etoricoxib was found to be associated with a statistically significantly lower rate of treatment discontinuation due to hepatotoxicity than diclofenac therapy. The treatment discontinuation rate in patients treated with etoricoxib was $0.3 \%$ and in patients treated with diclofenac was 2.7\%. Expressed per 100 patient-years, the index was 0.22 in the etoricoxib group and 1.84 in the diclofenac group [45].

According to the Summary of Product Characteristics, gastrointestinal contraindications to etoricoxib treatment include:

- active gastric and/or duodenal ulcers or gastrointestinal bleeding,

- inflammatory bowel disease,

- severe hepatic dysfunction.

\section{Cardiovascular safety of etoricoxib}

Treatment with all nonsteroidal anti-inflammatory drugs is associated with an elevated risk of cardiovascular complications. NSAIDs increase cardiovascular risk by elevating blood pressure and causing water and sodium retention (potentially leading to exacerbation of heart failure), inducing a prothrombotic effect and impairing the efficacy of concomitantly used acetylsalicylic acid. Patients treated with non-selective NSAIDs should take ASA at least 2 hours before the NSAID, otherwise the anti-platelet effect of ASA is markedly reduced or eliminated altogether. This is a considerable inconvenience for patients who, in the event of pain, usually fail to ensure an appropriate interval between taking ASA and NSAID. A good choice in such situations is a coxib drug which can be taken before or concurrently with
ASA. This is because coxibs are selective inhibitors of cyclooxygenase COX-2, whereas ASA inhibits cyclooxygenase COX-1 $[46,47]$.

Coxibs are generally regarded as more harmful for the cardiovascular system than non-selective COX-1 inhibitors, even though recent randomized studies have not provided evidence for this claim. The prospective multi-centre program MEDAL showed that the incidence of severe adverse effects in the form of thrombotic cardiovascular changes and cardiovascular death was similar in the group of patients with arthritis treated with etoricoxib and in the group receiving diclofenac. MEDAL was a study program including a pooled analysis of data from three large double-blind, randomized, controlled trials (MEDAL, EDGE and EDGE II). Overall, it involved nearly 35,000 patients with OA and RA who were treated for an average of 17.9 months. The participants of the program had a wide range of cardiovascular risk factors. The severity of cardiovascular risk associated with etoricoxib was shown to be correlated with the dose of the drug and the duration of treatment [45].

The results of several recently published meta-analyses and observational studies also point to the conclusion that etoricoxib has a similar safety profile to other NSAIDs [48-50]. One meta-analysis of results of randomized controlled and placebo-controlled trials demonstrated that the incidence of serious cardiovascular adverse effects in OA and RA patients treated with etoricoxib was comparable to placebo and naproxen [51].

Cardiovascular contraindications to etoricoxib therapy include:

- congestive heart failure (NYHA II-IV),

- inadequately controlled hypertension with BP persistently elevated above 140/90 $\mathrm{mmHg}$,

- ischemic heart disease, peripheral arterial disease and/or cerebrovascular disease.

Since the risk of cardiovascular complications associated with etoricoxib increases with dose and duration of therapy, the lowest effective daily dose should be used, and the duration of treatment should be as short as possible.

\section{Etoricoxib and other NSAIDs (selective and non-selective COX-2 inhibitors)}

In comparing etoricoxib with other NSAIDs, etoricoxib shows high analgesic potency, higher than celecoxib and comparable to diclofenac, and a better safety profile with regard to the upper gastrointestinal tract and lower hepatotoxicity compared to diclofenac [43]. However the risk of cardiovascular adverse effects is comparable to other NSAIDs, as shown in recent meta-analyses, ran- 
domized and observational studies [18, 30]. The increase in cardiovascular risk attributed to etoricoxib and other NSAIDs depends on the dose of the drug and the duration of therapy. Importantly, simultaneous use with acetylsalicylic acid (cardioprotective doses) without a reduction or elimination of the activity of ASA is possible.

\section{Conclusions}

Comparison of etoricoxib and other NSAIDs shows that the former is a beneficial therapeutic option in patients with osteoarthritis, rheumatoid arthritis, ankylosing spondylitis or gout who have few cardiovascular risk factors and, at the same time, a relatively high risk of gastrointestinal complications.

The article was compiled in cooperation with Sandoz.

The authors were lecturers and consultants of the company Sandoz.

\section{References}

1. Dallob A, Hawkey CJ, Greenberg H, et al. Characterization of etoricixib, a novel selective COX-2 inhibitor. J Clin Pharmacol 2003; 43: 573-585.

2. Leung GJ, Rainsford KD, Kean WF. Osteoarthritis of the hand I: aetiology and pathogenesis, risk factors, investigation and diagnosis. J Pharm Pharmacol 2013; 66: 339-346.

3. Bijsterbosch J, Meulenbelt I, Watt I, et al. Clustering of hand osteoarthritis progression and its relationship to progression of osteoarthritis at the knee. Ann Rheum Dis 2014; 73: 567-572.

4. Hochberg MC, Altman RD, April KT, et al. American College of Rheumatology 2012: Recommendations for the use of nonpharmacologic and pharmacologic therapies in osteoarthritis of hand, hip, and knee. Arthritis Care Res 2012; 64: 465-474.

5. Burmester G, Lanas A, Biasucci L, et al. The appropriate use of non-steroidal anti-inflammatory drugs in rheumatic disease: opinions of a multidisciplinary European expert panel. Ann Rheum Dis 2011; 70: 818-822.

6. Rahme E, Pettitt D, LeLorier J. Determinants and sequelae associated with utilization of acetaminophen versus traditional nonsteroidal antiinflammatory drugs in an elderly population. Arthritis Rheum 2002; 46: 3046-3054.

7. Van Laar M, Pergolizzi JV Jr, Mellinghoff HU, et al. Pain Treatment in Arthritis - Related Pain: Beyond NSAIDs. Open Rheumatol J 2012; 6: 320-330.

8. Puopolo A, Boice JA, Fidelholtz JL, et al. A randomized placebo-controlled trial comparing the efficacy of etoricoxib $30 \mathrm{mg}$ and ibuprofen $2400 \mathrm{mg}$ for the treatment of patients with osteoarthritis. Osteoarthritis and Cartilage 2007; 15: 1348-1356.

9. Bingham CO, Sebba Al, Rubin BR, et al. Efficacy and safety of etoricoxib $30 \mathrm{mg}$ and celecoxib $200 \mathrm{mg}$ in the treatment of osteoarthritis in two identically designed, randomized, placebo-controlled, non-inferiority studies. Rheumatology 2007; 46: 496-507.
10. Baraf HS, Fuentealba C, Greenwald M, et al. Gastrointestinal side effects of etoricoxib in patients with osteoarthritis: results of the Etoricoxib versus Diclofenac Sodium Gastrointestinal Tolerability and Effectiveness (EDGE) trial. J Rheumatol 2007; 34: 408-419.

11. Curtis SP, Bockow B, Fisher C, et al. Etoricoxib in the treatment of osteoarthritis over 52-weeks: a double-blind, active-comparator controlled trial [NCT00242489]. BMC Musculoskeletal Disorders 2005; 6: 58-68.

12. Stam W, Jansen J, Taylor S. Efficacy of etoricoxib, celecoxib, lumiracoxib, non-selective NSAIDs, and acetaminophen in osteoarthritis: a mixed treatment comparison. Open Rheumatol J 2012; 6: 6-20.

13. da Costa BR, Reichenbach S, Keller N, et al. Effectiveness of non-steroidal anti-inflammatory drugs for the treatment of pain in knee and hip osteoarthritis: a network meta-analysis. Lancet 2017; 390 (10090): e21-e33.

14. Smolen JS. EULAR recommendations for the management of rheumatoid arthritis with synthetic and biological disease-modifying antirheumatic drugs: 2016 update. Ann Rheum Dis 2017; 76: 960-977.

15. Collantes E, Curtis SP, Lee KW, et al. A multinational randomized, controlled, clinical trial of etoricoxib in the treatment of rheumatoid arthritis [ISRCTN25142273]. BMC Family Practice 2002; 3: 10.

16. Kvien TK, Greenwald M, Peloso PM, et al. Do COX-2 inhibitors provide additional pain relief and anti-inflammatory effects in patients with rheumatoid arthritis who are on biological disease-modifying anti-rheumatic drugs and/or corticosteroids? Post-hoc analyses from a randomized clinical trial with etoricoxib. BMC Musculoskeletal Disorders 2015; 16: 26.

17. Bickham K, Kivitz AJ, Mehta A, et al. Evaluation of two doses of etoricoxib, a COX-2 selective non-steroidal anti-inflammatory drug (NSAID), in the treatment of Rheumatoid Arthritis in a double-blind, randomized controlled trial. BMC Musculoskletal Disorders 2016; 17: 331-342.

18. Krueger K, Lino L, Dore R, et al. Gastrointestinal tolerability of etoricoxib in rheumatoid arthritis patients: results of the etoricoxib vs diclofenac sodium gastrointestinal tolerability and effectiveness trial (EDGE-II). Ann Rheum Dis 2008, 67: 315-322.

19. van der Heijde D, Ramiro S, Landewé R, et al. 2016 update of the ASAS-EULAR management recommendations for axial spondyloarthritis. Ann Rheum Dis 2017, 76: 978-991.

20. Wanders A, Heijde Dv, Landewé R, et al. Nonsteroidal antiinflammatory drugs reduce radiographic progression in patients with ankylosing spondylitis: a randomized clinical trial. Arthritis Rheum 2005, 52: 1756-1765.

21. Smolen JS, Braun J, Dougados M, et al. Treating spondyloarthritis, including ankylosing spondylitis and psoriatic arthritis, to target: recommendations of an international task force. Ann Rheum Dis 2014, 73: 6-16.

22. Tsai WC, Ou TT, Yen JH, et al. Long-term frequency use on non-steroidal anti-inflammatory drugs might protect patients with ankylosing spondylitis from cardiolvascular diseases: a nationwide case-control study. Plos One 2015; 10: e0126347.

23. Sieper J, Lenaerts J, Wollenhaupt J, et al. Maintenance of biologic-free remission with naproxen or no treatment in pa- 
tients with early, active axial spondyloarthritis: resulta from a 6-month, randomised, open-label study, INFAST Part2. Ann Rheum Dis 2014, 73: 108-113.

24. van der Heijde D, Baraf HS, Ramos-Remus C, et al. Evaluation of the efficacy of etoricoxib in ankylosing spondylitis: Results of a fifty-two-week, randomized, controlled study. Arthritis Rheum 2005; 52: 1205-1215.

25. Wang R, Dasgupta A, Ward MM. Comparative efficacy of non-steroidal anti-inflammatory drugs in ankylosing spondylitis: a Bayesian network meta-analysis of clinical trials. Ann Rheum Dis 2016, 75: 1152-1160.

26. Gratacós J, Moreno Martínez-Losa M, Font P, et al. Etoricoxib in ankylosing spondylitis: is there a role for active patients refractory to traditional NSAIDs? Clin Exp Rheumatol 2016, 34: 94-99.

27. Bae JM, Choo JY, Kim KJ, et al. Association of inflammatory bowel disease with ankylosing spondylitis and rheumatoid arthritis: A nationwide population-based study. Modern Rheumatology 2017; 27: 435-440.

28. Ribaldone DG, Fagoonee S, Astegiano M, et al. Coxib safety in patients with inflammatory bowel diseases: a meta-analysis. Pain Physician 2015; 18: 599-607.

29. Sheth T, Pitchumoni CS, Das KM. Management of musculoskeletal manifestation in inflammatory bowel disease. Gastroenterol Res Pract 2015; 2015: 387891.

30. Jansen JP, Gaugris S, Choy EH, et al. Cost effectiveness of etoricoxib versus celecoxib and non-selective NSAIDS in the treatment of ankylosing spondylitis. Pharmacoeconomics 2010; 28: 323-344.

31. Khanna D, Fitzgerald JD, Khanna PP, et al. 2012 American College of Rheumatology guidelines for management of gout. Part 1: systematic nonpharmacologic and pharmacologic therapeutic approaches to hyperuricemia. Arthritis Care Res 2012, 64: 1431-1446.

32. Khanna D, Khanna PP, Fitzgerald JD, et al. 2012 American College of Rheumatology guidelines for management of gout. Part 2: therapy and antiinflammatory prophylaxis of acute gouty arthritis. Arthritis Care Res 2012; 64: 1447-1461.

33. Richette P, Doherty M, Pascual E, et al. 2016 updated EULAR evidence-based recommendations for management of gout. Ann. Rheum. Dis 2017; 76: 29-42.

34. Kiltz U, Smolen J, Bardin T, et al. Treat-to-target (T2T) recommendations for gout. Ann Rheum Dis 2017, 76: 632-638.

35. Croom KF, Siddiqui MA. Etoricoxib. A review of its use in the symptomatic treatment of osteoarthritis, rheumatoid arthritis, ankylosing spondylitis and acute gouty arthritis. Cochrane Database Syst Rev 2014; 9: CD010120.

36. Zhang S, Zhang Y, Liu P, et al. Efficacy and safety of etoricoxib compared with NSAIDs in acute gout: a systematic review and a meta-analysis. Clin Rheumatol 2016; 35: 151-158.

37. Schlansky B, Hwang JH. Prevention of nonsteroidal anti-inflammatory drug-induced gastropathy. J Gastroenterol 2009; 44 Suppl. 19: 44-52.

38. Huang JQ, Sridhar S, Hunt RH. Role of Helicobacter pylori infection and non-steroidal anti-inflammatory drugs in peptic-ulcer disease: a meta-analysis. Lancet 2002; 359:14-22.
39. Peura DA, Lanza FL, Gostout CJ, et al. The American College of Gastroenterology Bleeding Registry: preliminary findings. Am J Gastroenterol 1997; 92: 924-928.

40. Bhatt DL, Scheiman J, Abraham NS, et al. ACCF/ACG/AHA 2008 expert consensus document on reducing the gastrointestinal risks of antiplatelet therapy and NSAID use: a report of the American College of Cardiology Foundation Task Force on Clinical Expert Consensus Documents. J Am Coll Cardiol 2008; 52: 1502-1517.

41. Garcia Rodríguez LA, Hernández-Díaz S. The risk of upper gastrointestinal complications associated with nonsteroidal anti-inflammatory drugs, glucocorticoids, acetaminophen, and combinations of these agents. Arthritis Res 2001; 3: 98-101.

42. Sturkenboom MC, Burke TA, Dieleman JP, et al. Underutilization of preventive strategies in patients receiving NSAIDs. Rheumatology (Oxford) 2003; 42 (suppl 3): iii23-iii31.

43. Laine L, Curtis SP, Cryer B, et al. Assessment of upper gastrointestinal safety of etoricoxib and diclofenac in patients with osteoarthritis and rheumatoid arthritis in the Multinational Etoricoxib and Diclofenac Arthritis Long-term (MEDAL) programme: a randomised comparison. Lancet 2007; 369: 465473.

44. Hunt RH, Harper S, Watson DJ, et al. The gastrointestinal safety of the COX-2 selective inhibitor etoricoxib assessed by both endoscopy and analysis of upper gastrointestinal events. Am J Gastroenterol 2003; 98: 1725-1733.

45. Cannon CP, Curtis SP, FitzGerald GA, et al. Cardiovascular outcomes with etoricoxib and diclofenac in patients with osteoarthritis and rheumatoid arthritis in the Multinational Etoricoxib and Diclofenac Arthritis Long-term (MEDAL) programme: a randomised comparison. Lancet 2006; 368: 1771-1781.

46. Farkouh ME, Greenberg JD, Jeger RV, et al. Cardiovascular outcomes in high risk patients with osteoarthritis treated with ibuprofen, naproxen or lumiracoxib. Ann Rheum Dis 2007; 66: 764-770.

47. Wilner KD, Rushing M, Walden C, et al. Celecoxib Does Not Affect the Antiplatelet Activity of Aspirin in Healthy Volunteers. J Clin Pharmacol 2002; 42: 1027-1030.

48. Trelle S, Reichenbach S, Wandel S, et al. Cardiovascular safety of non-steroidal anti-inflammatory drugs: network meta-analysis. BMJ 2011; 342: c7086.

49. Bhala N, Emberson J, Merhi A, et al.; Coxib and traditional NSAID Trialists' (CNT) Collaboration. Vascular and upper gastrointestinal effects of non-steroidal anti-inflammatory drugs: meta-analyses of individual participant data from randomised trials. Lancet 2013, 382: 769-779.

50. van Walsem A, Pandhi S, Nixon RM, et al. Relative benefit-risk comparing diclofenac to other traditional non-steroidal anti-inflammatory drugs and cyclooxygenase-2 inhibitors in patients with osteoarthritis or rheumatoid arthritis: a network meta-analysis. Arthritis Res Ther 2015, 17: 66

51. De Vecchis R, Baldi C, Di Biase G, et al. Cardiovascular risk associated with celecoxib or etoricoxib: a meta-analysis of randomized controlled trials which adopted comparison with placebo or naproxen. Minerva Cardioangiol 2014, 62: 437-448.

R/ETOR/005/11-2017 